\title{
Review
}

Michael J. Duffy*, Eleftherios P. Diamandis and John Crown

\section{Circulating tumor DNA (ctDNA) as a pan-cancer screening test: is it finally on the horizon?}

https://doi.org/10.1515/cclm-2021-0171

Received February 5, 2021; accepted March 29, 2021;

published online April 15, 2021

Abstract: The detection of cancer at an early stage while it is curable by surgical resection is widely believed to be one of the most effective strategies for reducing cancer mortality. Hence, the intense interests in the development of a simple pan-cancer screening test. Lack of sensitivity and specificity when combined with the low prevalence of most types of cancer types in the general population limit the use of most of the existing protein biomarkers for this purpose. Like proteins, tumor DNA also can be released into the circulation. Such circulating tumor DNA (ctDNA) can be differentiated from normal cell DNA by the presence of specific genetic alteration such as mutations, copy number changes, altered methylation patterns or being present in different sized fragments. Emerging results with test such as CancerSEEK or GRAIL suggest that the use of ctDNA can detect cancer with specificities $>99 \%$. Sensitivity however, is cancer type and stagedependent, varying from approximately $40 \%$ in stage I disease to approximately $80 \%$ in stage III disease. It is important to stress however, that most of the studies published to date have used patients with an established diagnosis of cancer while the control population were

\footnotetext{
*Corresponding author: Michael J. Duffy, Professor, UCD School of Medicine, Conway Institute of Biomolecular and Biomedical Research, University College Dublin, Dublin, Ireland; and UCD Clinical Research Centre, St. Vincent's University Hospital, Dublin, Ireland, Phone: +3531 7165814, Fax: +35312696018,

E-mail: michael.j.duffy@ucd.ie

Eleftherios P. Diamandis, Department of Laboratory Medicine and Pathobiology, University of Toronto, Toronto, Canada; Department of Pathology and Laboratory Medicine, Mount Sinai Hospital, Toronto, Canada; Department of Clinical Biochemistry, University Health Network, Toronto, Canada; and Lunenfeld-Tanenbaum Research Institute, Mount Sinai Hospital, Toronto, Canada. https://orcid.org/ 0000-0002-1589-820X

John Crown, Department of Medical Oncology, St Vincent's University Hospital, Dublin, Ireland
}

healthy individuals. Although the emerging results are promising, evidence of clinical utility will require demonstration of reduced mortality following evaluation in a prospective randomized screening trial.

Keywords: cancer; circulating; ctDNA; early diagnosis; pan-cancer; screening.

\section{Introduction}

The development of a simple, fast, accurate and inexpensive pan-cancer screening tests is one of the biggest challenges in cancer research. Achieving such a goal however, is likely to be highly rewarding, as it is widely believed that the detection of cancer at an early stage when it is curable by surgical resection is one of the most effective strategies for reducing cancer morbidity and mortality. Indeed, there is considerable evidence from studies with several different cancer types that early diagnosis results in improved outcome [1]. Even a reduction in cancer stage at first diagnosis has the potential to reduce death rates. Thus, Clarke et al. [2] calculated that if all stage IV cancers were diagnosed at stage III, this would result in a reduction of $15 \%$ of all cancer-related deaths.

Theoretically, a non/minimally invasive test such as a blood-based biomarker with high specificity for cancer, high sensitivity for early-stage tumors, an ability to preferentially detect clinically significant rather than indolent tumors and an ability to identify the site of origin of a cancer, might be expected to approach the ideal type of test for screening/early diagnosis. The identification and validation of such a biomarker however, presents several major challenges, as outlined below [3]. Although challenging, the first steps appear to have been recently made in the development of pan-cancer blood-based cancer screening tests. The aim of this article is to review these advances. Firstly, however, we briefly discuss the obstacles in the development of a pan-cancer screening test. 


\section{Challenges in the development of a screening/early diagnostic cancer biomarker}

One of the most important requirements of a cancer screening test is high specificity for malignancy which ideally should approach 100\% [4]. As the prevalence of a particular cancer type is relatively low in the general population $(<1 \%)$, high specificity is necessary to minimize possible harm due to false-positive findings [3]. Lack of specificity is a particular problem with existing protein biomarkers as exemplified by the use of PSA in screening for prostate cancer [5] and CA 125 in screening for ovarian cancer [6].

In addition to high specificity, high sensitivity is necessary to enable the detection of small/low volume tumors. Ideally, a screening test should detect cancer prior to the initiation of metastasis, i.e., when it is curable by surgical resection. Even, if a tumor has metastasized, it may still be possible to eradicate it using surgery in combination with adjuvant systemic treatment [7]. As with specificity, a major disadvantage of the current generation of protein biomarkers is that their concentrations are rarely increased in blood from patients with small localized tumors and only become elevated when distant metastasis is present. Indeed, if standard biomarkers are found to be elevated in patients with apparently localized cancer, it is likely to signify the presence of occult metastasis. Consequently, with a few possible exceptions (HCG for gestational trophoblastic disease/choriocarcinoma, PSA for prostate cancer in men 55-69 years of age, following a process of shared decision making and informed consistent), the available protein biomarkers are of little value in screening or detecting malignancy at an early or low volume stage.

A further requirement for a circulating screening/early diagnostic biomarker is the ability to identify the tissue of origin of a newly diagnosed malignancy. Otherwise, a positive biomarker test can lead to expensive and potentially time-consuming imaging investigations. With the exception of PSA and thyroglobulin, there are no other tissue-specific cancer biomarkers currently in clinical use.

Finally, the test should allow the detection of clinically significant (aggressive or likely to progress) early cancers and minimize the detection of indolent lesions. This is important to prevent overdiagnosis (diagnosis of cancers that would never cause harm if not detected) and possible overtreatment. Thus, ideally, an early diagnostic test should also have prognostic ability, i.e., be able to differentiate between indolent and aggressive cancers. It is important to state that overdiagnosis is a major limitation of most of the currently used cancer screening tests, as overall about $20 \%$ of five common cancers are estimated to be overdiagnosed by the process [8]. In the European randomized prospective trial for prostate cancer screening, approximately $50 \%$ of the screened-detected cancers were deemed to be overdiagnosed [5].

Other important criteria for a cancer screening/early detection test are listed in Table 1.

\section{Circulating tumor DNA (ctDNA)}

Essentially, cancer is a genetic disease defined by alterations at the nuclear DNA level. Alteration such as mutations, copy number changes, translocations and epigenetic modifications in key driver genes (e.g., oncogenes and tumor suppressor genes) are the primary causes of cancer. Altered DNA from these cancer-causing genes can be shed into the circulation and thus potentially used to detect cancer. Such tumor-derived DNA found in blood is commonly referred to as circulating tumor DNA (ctDNA). In most cancer patients, ctDNA constitutes a small proportion of the total circulating free DNA (cfDNA), especially in patients with early cancer (i.e., less than $0.5 \%$ ). ctDNA however, can be differentiated from normal cell-derived DNA by the presence of specific genetic alteration (mutations, different gene copy numbers, altered methylation patterns, presence of viral sequences). In addition, ctDNA fragments tend to exist in different molecular sizes compared to DNA derived from normal cells [9]. These differences between tumor-derived and normal cell-derived DNA are currently being actively investigated for the detection of early cancer (Table 2), see below.

\section{Use of ctDNA for cancer screening/ early detection}

\section{Assays based on mutation detection}

Using a highly sensitive sequencing method known as targeted error correction sequencing (TEC-Seq), Phallen et al. [10] compared the ctDNA mutational profile of 58 cancer-associated genes from 200 patients with an established diagnosis of stage I/II cancer and 44 healthy controls. Of 200 patients with cancer, 134 (67\%) had mutations detected in their ctDNA. However, for patients with stage I disease, mutations were detected in only 35/64 
Table 1: Ten criteria important for a cancer screening test and how ctDNA assays meet these criteria.

\begin{tabular}{|c|c|c|}
\hline Criteria & Why important & How ctDNA assays meets criteria \\
\hline gh specificity & $\begin{array}{l}\text { To minimize false-positives and thereby } \\
\text { reduce unnecessary work-up, overdiagnosis, } \\
\text { overtreatment }\end{array}$ & $\begin{array}{l}\text { Some assays (e.g., CancerSEK and Galleri) appear to have } \\
\text { high specificity using healthy subjects. Subjects with } \\
\text { benign/inflammatory disease have not so far been } \\
\text { investigated. CH-derived mutations may reduce specificity }\end{array}$ \\
\hline High sensitivity & $\begin{array}{l}\text { To detect early tumors that may be curable by } \\
\text { surgery }\end{array}$ & $\begin{array}{l}\text { Variable, depending on tumor type and tumor stage. Most } \\
\text { studies used patients with a previous diagnosis of cancer. } \\
\text { Sensitivity is likely to be less in asymptomatic subjects }\end{array}$ \\
\hline High PPV & $\begin{array}{l}\text { To reduce unnecessary work-up, over- } \\
\text { diagnosis, overtreatment }\end{array}$ & $\begin{array}{l}\text { Some assays may be acceptable for high-prevalent can- } \\
\text { cers but not for low-prevalent cancers. PPV could be } \\
\text { improved by focusing on high-risk individuals }\end{array}$ \\
\hline High NPV & $\begin{array}{l}\text { To reduce the probability of missing poten- } \\
\text { tially curable tumors }\end{array}$ & $\begin{array}{l}\text { Existing assays may miss low-stage tumors and brain } \\
\text { tumors }\end{array}$ \\
\hline Ability to localize site of tumor & To minimize imaging for localizing tumor site & $\begin{array}{l}\text { Variable, assays based on altered methylation } \\
\text { (e.g., Galleri) may be better that those detecting mutations }\end{array}$ \\
\hline Ability to decrease mortality & Key endpoint for a cancer screening test & Not yet shown, trials in progress \\
\hline $\begin{array}{l}\text { Availability of an analytically } \\
\text { validated test and validated } \\
\text { pre-analytical procedure }\end{array}$ & $\begin{array}{l}\text { To ensure consistency of measurement over } \\
\text { time and across different sites }\end{array}$ & $\begin{array}{l}\text { Little information published on analytical performance of } \\
\text { ctDNA assays }\end{array}$ \\
\hline Availability of an non-invasive test & To ensure wide uptake & ctDNA assays are relatively non-invasive \\
\hline $\begin{array}{l}\text { Availability of a non-inexpensive } \\
\text { test }\end{array}$ & To ensure wide use across the world & $\begin{array}{l}\text { ctDNA assays are relatively expensive, more expensive } \\
\text { than protein biomarkers }\end{array}$ \\
\hline $\begin{array}{l}\text { Ability to minimize overdetection/ } \\
\text { overtreatment of indolent cancers }\end{array}$ & $\begin{array}{l}\text { To avoid unnecessary work-up and treatment } \\
\text { of non-life threatening tumors }\end{array}$ & Unclear, data not yet available \\
\hline
\end{tabular}

$\mathrm{CH}$, hematopoiesis; PPV, positive predictive value; NPV, negative predictive value.

Table 2: ctDNA tests under investigation for pan-cancer screening/ early detection of cancer.

\begin{tabular}{llr}
\hline Test & Parameters detected & Refs \\
\hline TEC-Seq & Mutations & 10 \\
CancerSEEK & Mutated DNA + eight standard biomarkers & 11 \\
GRAIL & Altered DNA methylation & 20 \\
cfMeDIP-seq & Altered DNA methylation & $22-24$ \\
PanSeer & Altered DNA methylation & 21 \\
DELFI & Altered DNA fragment patterns & 27 \\
\hline
\end{tabular}

TEC-Seq, targeted error correction sequencing; cfMeDIP-seq, cell-free methylated DNA immunoprecipitation and high-throughput

sequencing; DELFI, DNA evaluation of fragments for early interception.

(54\%) of cases. Sensitivity was highest in patients with colorectal cancer (83\%), followed by ovarian cancer (71\%), lung cancer $(62 \%)$ and breast cancer (56\%). For patients with stages I or II disease, sensitivity was $71 \%$ in patients with CRC, $68 \%$ in ovarian cancer and 59\% in both lung and breast cancer. Importantly, there were no mutations detected in driver genes associated with solid cancer (genes responsible for driving tumor cell growth) in any of 44 healthy controls investigated. However, $16 \%$ of healthy controls had mutations in genes associated with a benign condition known as clonal hematopoiesis of indeterminate potential (CHIP) (see below).
In an attempt to further enhance sensitivity, Cohen et al. [11] developed a combined assay that detects both DNA mutations and protein biomarkers. This test, known as CancerSEEK, detects mutations at 2,001 locations across 16 cancer-associated genes as well as the concentration of eight protein biomarkers. In a retrospective case-control study that included 1,005 patients with clinically detected cancers of the ovary, liver, stomach, pancreas, esophagus, colorectum, lung, or breast. CancerSEEK was found to be positive in a median of $70 \%$ of cases. Similar to the situation with standard protein biomarkers, sensitivity increased with increasing stage of the cancer, i.e., median sensitivity was 43,73 and $78 \%$ for patients with stages I, II and III disease, respectively. Sensitivity was highest in patients with ovarian or liver cancer (98\%) and lowest in breast cancer (33\%). For patients with stage I disease, sensitivity was $100 \%$ in those with liver cancer but was only $20 \%$ in those with esophageal cancer. Specificity was $>99 \%$, i.e., only $7 / 812$ apparently healthy controls gave a positive result. Some of these subjects may subsequently have developed cancer but this does not appear to have been investigated. In the 626 patients who tested positive, use of a machine learning algorithm that include the CancerSEEK analytes and patient sex narrowed the tumor location to either of two organs in $83 \%$ of patients and 
correctly located the specific site in $68 \%$ of cases. In an update on CancerSEEK, Douville et al. [12] showed that a combination of aneuploidy (copy number changes) with mutation detection and the eight protein biomarkers yielded a median sensitivity of $80 \%$ for cancer detection, while only $1 \%$ of 812 healthy subjects tested positive.

An earlier version of the CancerSEEK test in combination with PET-CT imaging, was recently investigated in a prospective exploratory study known as DETECT-A [13]. Of 9,911 asymptomatic women screened, 490 (4.9\%) gave a positive result in the initial baseline blood test. Following exclusion of CHIP-derived mutations, 134 subjects (1.35\% of the 9,911 participants) had their initial result confirmed in a follow-up test. Of these 134 women, 127 underwent PET-CT scans or other imaging procedures. Resulting from these tests, 26 cancers were detected of which 17 (65\%) were described as having early disease. In addition to these 26 cases detected using ctDNA, 24 further cancers were detected by standard screening tests and 46 by other diagnostic modalities during the study. Overall, the ctDNA test had a sensitivity of $27.1 \%$, a specificity of $98.9 \%$, a positive predictive value (PPV) of $19.4 \%$ and a negative predictive value (NPV) of $99.3 \%$. Combining the ctDNA test with any form of imaging increased specificity to $99.6 \%$ and the PPV to $40.6 \%$, but had little impact on the sensitivity or NPV. It should be stated that this study was not designed to determine whether detecting and treating early cancer reduced mortality but to show that ctDNA testing was feasible and could be safely used to detect early cancer in asymptomatic subjects.

In 2019, CancerSEEK received Breakthrough Device designation from the U.S. Food and Drug Administration for the detection of genetic mutations and proteins associated with pancreatic and ovarian cancers (https://hub.jhu.edu/ 2019/06/03/cancerseek-blood-test/). Further validation of CancerSEEK is ongoing in a prospective, observational study (ASCEND) involving 1,000 subjects with known or suspected cancer and 2,000 subjects with no evidence of cancer (ClinicalTrials.gov Identifier: NCT04213326). The primary objective of this study is to develop and validate the CancerSEEK test for the early detection of cancer.

\section{Assays based on altered methylation patterns}

Compared to normal healthy tissue, DNA in tumors exhibits aberrant methylation. In particular, there is a progressive gain of promoter $\mathrm{CpG}$-island hypermethylation and a loss of $\mathrm{CpG}$ methylation in non-CpG-island promoters $[14,15]$. In at least some situations, these alterations occur early in the formation of malignancy and appear to be tumor-type specific $[14,15]$. As with mutant DNA, aberrantly methylated DNA can be detected in blood and thus potentially used for the early detection of cancer [16]. Advantages of measuring methylation alterations over mutations include greater abundance and enhanced tissue-specificity [17-19]. The enhanced tissue-specificity should make methylation-based assays superior to mutation detection for the identification of the tissue of origin of newly-detected cancers.

One of the most high profile although not necessary the most accurate plasma methylation tests for early cancer detection is the GRAIL test (also known as the Galleri test). This test uses targeted whole genomic bisulfite sequencing of plasma DNA and machine learning to analyze a panel of $>100,000$ informative methylation loci. GRAIL has been investigated in a prospective multicentre case-control study containing 6,689 individuals (2,482 patients with cancer and 4,207 apparently healthy subjects) [20]. The 6,689 patients were divided into a training $(n=4,720)$ and an independent validation set $(n=1,969)$. In the validation set, the overall sensitivity of the test in 12 pre-specified cancer types for patients with stages I to III disease was $67.3 \%$. Positivity increased with increasing cancer stage, i.e., $39 \%$ in stage I, $69 \%$ in stage II, $83 \%$ in stage III, and $92 \%$ in stage IV. Specificity was $99.3 \%$, i.e., a $0.7 \%$ falsepositive rate. In contrast to the 12 pre-specified cancer types, sensitivity across a broader panel of more than 50 different cancer types was $18 \%$ in stage I, $43 \%$ in stage II, $81 \%$ in stage III, and $93 \%$ in stage IV. In patients with a diagnosed cancer, the tissue of origin was correctly localized in $93 \%$ of cases.

This test is undergoing further validation in several different clinical trials. One of these trials (known as STRIVE) is a prospective multi-center observational cohort study aiming to validate GRAIL for the early detection of breast and other cancers (ClinicalTrials.gov Identifier: NCT03085888). Another trial (SUMMIT) involves its validation for detecting multiple cancers at an early stage (NCT03934866). The third trial (The PATHFINDER) is a prospective, multi-center study assessing the possible implementation of the test into clinical practice (ClinicalTrials.gov Identifier: NCT04241796). In addition to these trials, it is planned to pilot the Galleri test in 165,000 subjects in England (140,000 people aged 50-79 years who have no signs or symptoms of cancer and 25,000 people aged 40 years and above with symptoms of cancer (https://www.england.nhs.uk/2020/11/nhs-topilot-potentially-revolutionary-blood-test/).

The GRAIL test was recently granted an US FDA breakthrough device designation (http://www.pmlive. 
com/pharma_news/grails_multi-cancer_blood_test_gets_ fda_breakthrough_status_1287854). It is important to state however that the Galleri test is intended to be complementary to, and not a replacement for U.S. guideline-recommended cancer screening tests.

A different plasma DNA methylation test, known as PanSeer, targets 10,613 CpG methylation sites across 477 genomic regions [21]. In a study involving 113 patients with an established diagnosis of cancer and 207 healthy controls, PanSeer was positive in $88 \%$ of patients at $96 \%$ specificity. Sensitivity was claimed to be similar in patients with early and late stage cancers. In 191 asymptomatic subjects who later developed one of five different cancers (stomach, esophageal, liver, CRC, lung), the test had a sensitivity of $95 \%$ at $95 \%$ specificity. Positivity was found up to 4 years in advance of diagnosis.

A further ctDNA methylation test, known as cfMeDIP (cell-free methylated DNA immunoprecipitation and highthroughput), uses affinity capture methods to enrich plasma DNA for densely methylated $\mathrm{CpG}$ regions of the genome. Following sequencing of the captured fragments of DNA, cfMeDIP employs machine-learning algorithms to differentiate patients with cancer from healthy controls [22]. To date, cfMeDIP has been mostly investigated in patients with renal and brain cancer. In a study involving 99 patients with renal cancer and 28 healthy controls, the test gave an AUROC (area under the receiver operating characteristic) curve of 0.99 [23]. For differentiating between patients with renal and bladder cancers $(n=21)$, the AUROC was 0.97. In a further study, cfMeDIP was found to differentiate between patients with gliomas $(\mathrm{n}=59)$ and those with other cancer types or healthy controls $(n=388)$ with an AUROC of 0.99 [24]. Furthermore, the test was able to distinguish between different types of brain tumors that can look similar on imaging such as gliomas, meningiomas, glial-neuronal tumors and brain metastases from systemic cancer.

\section{Assays based on different ctDNA fragment size}

While plasma DNA fragments derived from normal cells shows a predominant (modal) peak size of $167 \mathrm{bp}$, ctDNA tends to exist in both shorter (90-150 bp) and longer fragment sizes (250-300 bp) [25, 26]. Mouliere et al. [25] were one of the first to exploit this difference in fragment size to differentiate between patients with cancer and healthy controls. By selecting fragments between 90 and $150 \mathrm{bp}$, these authors were able to identify clinically important mutations and copy number alterations that would have been missed in the absence of sized-based separation. Integrating fragment length and copy number analysis of plasma DNA, led to a greater number of metastatic cancer detected, i.e., area under the curve (AUC) was $>0.99$, compared to AUC $<0.80$ without incorporating the fragmentation patterns.

Subsequently, Cristiano et al. [27] exploited this fragment size difference in an attempt to develop an early diagnostic test for cancer. The test known as DELFI (DNA evaluation of fragments for early interception) uses whole genomic sequencing and artificial intelligence to detect different patterns of DNA fragmentation in plasma from healthy controls and cancer patients. Using low coverage sequencing, the diagnostic potential of DELFI was investigated in 208 patients with seven different cancer types and in 215 apparently healthy individuals. Following the application of a machine-learning model that combined DNA fragmentation patterns and copy number changes, the test was found to differentiate between healthy subjects and patients with different cancer types with an overall sensitivity of $73 \%$ at $98 \%$ specificity. Sensitivity varied from $68 \%$ in stage 1 disease to $79 \%$ in those with stage III disease. Lowest sensitivity was found in breast cancer (57\%) and highest in lung cancer (100\%). The addition of ctDNA mutations to the DELFI data increased the sensitivity for cancer detection from 73 to $91 \%$, while maintaining similar specificity. The test however, was poor in correctly identifying the tissue-of-origin of the cancers, i.e., it was able to identify the tissue of origin to one of two sites in only $75 \%$ of cases.

\section{Critique of above findings}

\section{Specificity of ctDNA tests}

Compared with existing protein biomarkers, tumorderived DNA might be expected to be more cancerspecific. Indeed, the emerging results with Cancer SEEK and GRAIL mentioned above, reported specificities of $>99 \%$ using samples from apparently healthy controls. This high specificity may initially appear impressive and although no direct comparisons were made, it is likely to be superior to that available from standard protein biomarkers.

Although mutant DNA might be expected to be rarely detected in the circulation of healthy subjects, certain benign lesions, especially CHIP, can release mutant DNA into the circulation $[28,29]$. CHIP involves the clonal expansion of white blood cells and occurs in approximately $10-20 \%$ of apparently healthy subjects over 70 
years of age [30]. Using deep sequencing, Razavi et al. [31] reported that most of the DNA mutations in blood whether form healthy control subjects or patients with cancer had characteristics of been derived from CHIP ( $81.6 \%$ in controls subjects and 52\% in patients with malignancy). In another study, CHIP-derived mutations were detected in $76 \%$ of patients with lung cancer and in $91 \%$ of highrisk controls [32]. In this study the VAFs (the relative frequency of a variant/mutation expressed as a percentage) of the mutations detected in plasma and leukocytes were significantly correlated, suggesting a blood cell origin for the detected mutations. CHIP mutations with the highest VAF occurred in DNMT3A, TP53, TET2, ASXL1, PPM1D and IDH1/2 [28].

To overcome the problem of CHIP-derived mutations leading to false-positive findings, several strategies have been proposed. These include parallel measurement of white cell DNA or performing sequencing on size-based selection of circulating DNA fragments [32, 33]. As mentioned above, in the DETECT-A study [13], mutations related to $\mathrm{CH}$ were excluded by simultaneously sequencing DNA from the white blood cells. Performing these additional procedures however, is costly and time-consuming, thus limiting the attraction of ctDNA as a population-based screening test for cancer.

As well CHIP, mutations have been detected in several other benign lesions and even in normal tissues [34, 35]. Thus, following a comprehensive analysis of approximately 6,700 normal samples across 29 normal tissues, Iizhak et al. [34] identified multiple somatic mutations in a range of different apparently normal tissues, especially in sun-exposed skin, esophagus, and lung. These mutations tended to accumulate over both time and number of cell divisions. Clearly, therefore, DNA mutations are not unique to malignant tissue $[34,35]$ and indeed may be the "new normal" [34].

These findings beg the question whether benign lesions other than $\mathrm{CH}$ or even healthy individuals release mutant DNA into the circulation. The answer to this question appears to be yes. For example, in a study involving subjects from Sub-Saharan Africa where that the hepatocarcinogen, aflatoxin contaminates much of the traditional diet, mutation in TP53 were detected in 15\% of patients with liver cirrhosis and in 3\% of apparently healthy individuals [36]. In another study, mutations in KRAS or TP53 were found in plasma from apparently healthy subjects in 13/1,098 (1.2\%) and in 20/550 (3.6\%), respectively. Of these mutation-positive subjects, 16 developed cancer, on average after 18.3 months of followup [37].

\section{Sensitivity of ctDNA tests}

As well as high specificity, high sensitivity for early stage tumors is an important criterion for a cancer screening test. As with standard protein biomarkers, the sensitivity of ctDNA varies with cancer stage and site of origin. Thus, in the studies reported to date, sensitivity increased with increasing stage, i.e., from approximately $40 \%$ in stage I to approximately $80 \%$ in stage III malignancy $[12,20]$. The most likely reason for the greater sensitivity with higher stage disease is that increasing amounts of mutant DNA are shed into the circulation as the tumor size/volume enlarges. In addition to stage, sensitivity depends on the cancer type. Thus, using plasma from 21,807 patients with advanced tumor of diverse origins, the prevalence of ctDNA ranged from $51 \%$ in glioblastoma to $93 \%$ in small cell lung cancer [38]. As regards, levels of ctDNA, these were highest in CRC and lowest in gliomas [38].

Similar to the situation with specificity, direct comparisons of the comparative sensitivity of ctDNA and standard biomarkers have not been performed. Nevertheless, the above preliminary findings suggest that ctDNA has enhanced sensitivity compared to existing protein biomarkers, at least for some tumors. For example, the CancerSeek test was positive in $98 \%$ of patients with ovarian cancer at a specificity of $>99 \%$ [11]. This accuracy of CancerSeek appears to be higher than that of the standard biomarker for ovarian cancer, i.e., CA 125 [39]. For example, following a systematic review of the literature followed by a meta-analysis, Ferraro et al. [38] calculated that CA 125 had an overall sensitivity of $79 \%$ and specificity of $78 \%$ for ovarian cancer. The GRAIL test in patients with pancreatic cancer which was reported to be positive in $63 \%$ of patients with stage I disease, $83 \%$ in stage II, $75 \%$ in stage III and $100 \%$ in stage IV [20], also appears to be more sensitive than the standard biomarker, for pancreatic cancer, i.e., CA 19-9 [40]. However, neither CA 125 for ovarian cancer or CA 19-9 for pancreatic when used alone can be recommended as screening tests for their respective cancers because of their low prevalence and thus low PPV in asymptomatic subject (see below).

While the emerging but indirect data suggests that ctDNA has superior sensitivity than established biomarkers for detecting cancer, it ability to detect stage I disease $(<2 \mathrm{~cm})$ is limited, i.e., a sensitivity of $43 \%$ with CancerSEEK [11] and 39\% with GRAIL [20]. Potential strategies to improve sensitivity include drawing larger volumes of blood (not always feasible), using ultra-low sensitive sequencing, increasing the number of mutations analyzed, combining different types of genetic alterations 
(mutations, copy number changes and methylation patterns), combining ctDNA measurements with protein biomarkers (as in CancerSEEK) or enriching for low molecular weight DNA fragments prior to mutational analysis [25-27]. Again, these additional requirements limit the attractiveness of ctDNA assays for cancer screening.

Although potential strategies are available for enhancing sensitivity, calculations by Fiala and Diamandis [41, 42] indicated that ctDNA has a low probability of detecting tumors with a diameter of $\leq 10 \mathrm{~mm}$ using $10 \mathrm{ml}$ of whole blood. For tumors with a diameter of $\leq 6 \mathrm{~mm}$, these authors concluded that it was not possible to detect ctDNA in $10 \mathrm{ml}$ of blood, irrespective of the sensitivity or reliability of the assay used [41, 42]. At this small size, some tumors may already have metastasized and thus be potentially incurable by surgery but they might be curable using surgery followed by adjuvant therapy, especially if the amount of metastasis is minimal.

\section{Importance of PPV and disease prevalence for use of a cancer screening test}

While high specificity and high sensitivity are important requirements for any screening test applied in asymptomatic subjects, a further important criterion is its PPV. The PPV of a test and thus its potential clinical utility critically depend on the prevalence of the disease within the population undergoing investigation. Fiala and Diamandis have calculated the PPV of a theoretical screening test for 12 different cancer types assuming the test had a sensitivity of $30 \%$ and a specificity of $99 \%$ $[43,44]$. The $30 \%$ sensitivity level was selected as the authors concluded that this was the average reported value for patients with stages I and II disease in the GRAIL study discussed above. For most of the 12 cancers selected, the calculated PPV values were less than $10 \%$, varying from $1 \%$ for esophageal cancer to $30 \%$ for colorectal cancer. For relatively rare cancers that have high mortality rates such as pancreatic cancer and ovarian cancer, the respective PPV were 2.2 and $8.3 \%$.

As regards the PPV of 8.3 for ovarian cancer, it should be stated that it has been long advocated that any screening strategy for this disease should have a minimum PPV of $10 \%$ in order to limit the number of unnecessary surgeries [45]. To achieve this PPV value, a screening strategy should have a sensitivity of $\geq 75 \%$ and a specificity of at least $99.6 \%$ [45]. Interestingly, the two-stage risk of ovarian cancer algorithm used in the UK Collaborative Trial of Ovarian Cancer Screening trial which combines measurement of CA 125 with transvaginal ultrasound achieved a sensitivity of $85.8 \%$ and a specificity of 99.8\% [46]. Whether a ctDNA can match these values in asymptomatic women remains to be established.

The limitation imposed by the low PPV however, could be minimized by focusing on high-risk subjects for cancer such as heavy smokers, individual genetically predisposed to malignancy or immunocompromised individuals. With such high-risk subjects, the prevalence of occult malignancy would be expected to be higher than that in the general population. Hence, the PPV of a screening test would also be increased. A further setting in which ctDNA tests might be expected to have a higher PPV and thus clinical utility would be in symptomatic subjects (subjects with weight loss, abdominal pain, change in bowel habit, bleeding). Indeed, in this situation accelerating diagnosis might be expected to result in an improved outcome as increased time to diagnosis and treatment was found to be associated with poor outcome in several different cancer types [47]. A relatively noninvasive test in such symptomatic patients could minimize the number of expensive imaging or invasive endoscopy procedures, especially if it could localize the site of the cancer.

\section{Conclusions}

Clearly, the evaluation of ctDNA as a screening test for cancer is currently a highly active area of research. Currently, this research is best described as work in progress. It is important to state however, that most of the published reports to date have been carried out using patients with a previous diagnosis of the disease. In population screening, these metrics might be lower as sensitivities in asymptomatic populations might be expected to be less that in patients with clinically detected cancer while specificities might be less when subjects with benign conditions are analyzed.

While comparing patients with clinically detected cancer against healthy controls is a reasonable early step in evaluating new cancer screening tests, it does not provide proof of clinical utility. The latter will require evidence of a significant reduction in mortality from cancer based on a prospective randomized trial involving a screened and a control group of asymptomatic subjects. In these trials, it will important to show that use of a ctDNA screening 
test does not lead to the major overdetection and thus of potential overtreatment of indolent tumors and is costeffective. As mentioned above, prospective studies are currently ongoing with CancerSEEK and GRAIL. Their results are eagerly awaited. If positive, they have the potential to be a major step forward in reducing mortality from cancer.

Acknowledgments: The authors thank the Cancer Clinical Research Fund for funding this work.

Research funding: This research was funded by Cancer Clinical Research Fund.

Author contributions: MJD conceived the study and wrote the initial draft. EPD and JC edited the manuscript and suggested the inclusion of additional data. All authors have accepted responsibility for the entire content of this manuscript and approved its submission.

Competing interests: Authors state no conflict of interest with any product or entity mentioned in the article.

Informed consent: Not applicable.

Ethical approval: Not applicable.

\section{References}

1. Cho H, Mariotto AB, Schwartz LM, Luo J, Woloshin S. When do changes in cancer survival mean progress? The insight from population incidence and mortality. J Natl Cancer Inst Monogr 2014;49:187-97.

2. Clarke CA, Hubbell E, Kurian AW, Colditz GA, Hartman AR, Gomez SL. Projected reductions in absolute cancer-related deaths from diagnosing cancers before metastasis, 2006-2015. Cancer Epidemiol Biomarkers Prev 2020;29:895-902.

3. Aravanis AM, Lee M, Klausner RD. Next-generation sequencing of circulating tumor DNA for early cancer detection. Cell 2017;168: 571-4.

4. Barratt A, Mannes P, Irwig L, Trevena L, Craig J, Rychetnik L. Cancer screening. J Epidemiol Community Health 2002;56:899-902.

5. Schröder FH, Hugosson J, Roobol MJ, Tammela TL, Zappa M, Nelen $V$, et al. Screening and prostate cancer mortality: results of the European randomised study of screening for prostate cancer (ERSPC) at 13 years of follow-up. Lancet 2014;384:2027-35.

6. Buys SS, Partridge E, Black A, Johnson CC, Lamerato L, Isaacs C, et al. Effect of screening on ovarian cancer mortality: the prostate, lung, colorectal and ovarian (PLCO) cancer screening randomized controlled trial. J Am Med Assoc 2011;305:2295-303.

7. Pashayan N, Pharoah PDP. The challenge of early detection in cancer. Science 2020;368:589-90.

8. Glasziou PP, Bell KJ, Barratt AL. Estimating the magnitude of cancer overdiagnosis in Australia. Med J Aust 2020;213:189-189.e1.

9. Heitzer E, Haque IS, Roberts CES, Speicher MR. Current and future perspectives of liquid biopsies in genomics-driven oncology. Nat Rev Genet 2019;20:71-88.
10. Phallen J, Sausen M, Adleff V, Leal A, Hruban C, White J, et al. Direct detection of early-stage cancers using circulating tumor DNA. Sci Transl Med 2017;9:eaan2415.

11. Cohen JD, Li L, Wang Y, Thoburn C, Afsari B, Danilova L, et al. Detection and localization of surgically resectable cancers with a multi-analyte blood test. Science 2018;359:926-30.

12. Douville C, Cohen JD, Ptak J, Popoli M, Schaefer J, Silliman N, et al. Assessing aneuploidy with repetitive element sequencing. Proc Natl Acad Sci U S A 2020;117:4858-63.

13. Lennon AM, Buchanan AH, Kinde I, Warren A, Honushefsky A, Cohain AT, et al. Feasibility of blood testing combined with PET-CT to screen for cancer and guide intervention. Science 2020;369: eabb9601.

14. Baylin SB, Jones PA. Epigenetic determinants of cancer. Cold Spring Harb Perspect Biol 2016;8:a019505.

15. Feinberg AP. The key role of epigenetics in human disease prevention and mitigation. N Engl J Med 2018;378:1323-34.

16. Leal A, Sidransky D, Brait M. Tissue and cell-free DNA-based epigenomic approaches for cancer detection. Clin Chem 2020; 66:105-16.

17. Lehmann-Werman R, Neiman D, Zemmour H, Moss J, Magenheim J, Vaknin-Dembinsky A, et al. Identification of tissue-specific cell death using methylation patterns of circulating DNA. Proc Natl Acad Sci U S A 2016;113:E1826-34.

18. Guo S, Diep D, Plongthongkum N, Fung HL, Zhang K, Zhang K. Identification of methylation haplotype blocks aids in deconvolution of heterogeneous tissue samples and tumor tissue-of-origin mapping from plasma DNA. Nat Genet 2017;49: 635-42.

19. Fernandez AF, Assenov Y, Martin-Subero Jl, Balint B, Siebert R, Taniguchi $\mathrm{H}$, et al. A DNA methylation fingerprint of 1628 human samples. Genome Res 2012;22:407-19.

20. Liu MC, Oxnard GR, Klein EA, Swanton C, Seiden MV, CCGA Consortium. Sensitive and specific multi-cancer detection and localization using methylation signatures in cell-free DNA. Ann Oncol 2020;31:745-59.

21. Chen X, Gole J, Gore A, He Q, Lu M, Min J, et al. Non-invasive early detection of cancer four years before conventional diagnosis using a blood test. Nat Commun 2020;11:3475.

22. Shen SY, Burgener JM, Bratman SV, De Carvalho DD. Preparation of cfMeDIP-seq libraries for methylome profiling of plasma cellfree DNA. Nat Protoc 2019;14:2749-80.

23. Nuzzo PV, Berchuck JE, Korthauer K, Spisak S, Nassar AH, Abou Alaiwi $S$, et al. Detection of renal cell carcinoma using plasma and urine cell-free DNA methylomes. Nat Med 2020;26: 1041-3. Erratum in: Nat Med. 2020;26:1663.

24. Nassiri F, Chakravarthy A, Feng S, Shen SY, Nejad R, Zuccato JA, et al. Detection and discrimination of intracranial tumors using plasma cell-free DNA methylomes. Nat Med 2020;26:1044-7.

25. Mouliere F, Chandrananda D, Piskorz AM, Moore EK, Morris J, Ahlborn LB, et al. Enhanced detection of circulating tumor DNA by fragment size analysis. Sci Transl Med 2018;10:eaat4921.

26. Liu X, Lang J, Li S, Wang Y, Peng L, Wang W, et al. Fragment enrichment of circulating tumor DNA with low-frequency mutations. Front Genet 2020;11:147.

27. Cristiano S, Leal A, Phallen J, Fiksel J, Adleff V, Bruhm DC, et al. Genome-wide cell-free DNA fragmentation in patients with cancer. Nature 2019;570:385-9. 
28. Chan HT, Chin YM, Nakamura Y, Low SK. Clonal hematopoiesis in liquid biopsy: from biological noise to valuable clinical implications. Cancers (Basel) 2020;14:2277.

29. Fiala C, Diamandis EP. Circulating tumor DNA (ctDNA) is not a good proxy for liquid biopsies of tumor tissues for early detection. Clin Chem Lab Med 2020;58:1651-3.

30. Bauml J, Levy B. Clonal hematopoiesis: a new layer in the liquid biopsy story in lung cancer. Clin Cancer Res 2018;24: 4352-4.

31. Razavi P, Li BT, Brown DN, Jung B, Hubbell E, Shen R, et al. Highintensity sequencing reveals the sources of plasma circulating cell-free DNA variants. Nat Med 2019;25:1928-37.

32. Chabon JJ, Hamilton EG, Kurtz DM, Esfahani MS, Moding EJ, Stehr $\mathrm{H}$, et al. Integrating genomic features for non-invasive early lung cancer detection. Nature 2020;580:245-51.

33. Keller L, Belloum Y, Wikman H, Pantel K. Clinical relevance of blood-based ctDNA analysis: mutation detection and beyond. $\mathrm{Br}$ J Cancer 2021;124:345-58.

34. Yizhak K, Aguet F, Kim J, Hess JM, Kübler K, Grimsby J, et al. RNA sequence analysis reveals macroscopic somatic clonal expansion across normal tissues. Science 2019;364:eaaw0726.

35. Fiala C, Diamandis EP. Mutations in normal tissues-some diagnostic and clinical implications. BMC Med 2020;18:283.

36. Kirk GD, Lesi OA, Mendy M, Szymañska K, Whittle H, Goedert JJ, et al. 249(ser) TP53 mutation in plasma DNA, hepatitis B viral infection, and risk of hepatocellular carcinoma. Oncogene 2005; 24:5858-67.

37. Gormally E, Vineis P, Matullo G, Veglia F, Caboux E, Le Roux E, et al. TP53 and KRAS2 mutations in plasma DNA of healthy subjects and subsequent cancer occurrence: a prospective study. Cancer Res 2006;66:6871-6.
38. Zill OA, Banks KC, Fairclough SR, Mortimer SA, Vowles JV, Mokhtari R, et al. The landscape of actionable genomic alterations in cell-free circulating tumor DNA from 21,807 advanced cancer patients. Clin Cancer Res 2018;24:3528-38.

39. Ferraro S, Braga F, Lanzoni M, Boracchi P, Biganzoli EM, Panteghini M. Serum human epididymis protein 4 vs carbohydrate antigen 125 for ovarian cancer diagnosis: a systematic review. J Clin Pathol 2013;66:273-81.

40. Hasan S, Jacob R, Manne U, Paluri R. Advances in pancreatic cancer biomarkers. Oncol Rev 2019;13:410.

41. Fiala C, Diamandis EP. New approaches for detecting cancer with circulating cell-free DNA. BMC Med 2019;17:159.

42. Fiala C, Diamandis EP. Can a broad molecular screen based on circulating tumor DNA aid in early cancer detection?. J Appl Lab Med 2020;5:1372-7.

43. Fiala C, Diamandis EP. A multi-cancer detection test: focus on the positive predictive value. Ann Oncol 2020;31:1267-8.

44. Fiala C, Diamandis EP. Can a broad molecular screen based on circulating tumor DNA aid in early cancer detection? J Appl Lab Med 2020;5:1372-7.

45. Jacobs I, Bast RC Jr. The CA 125 tumour-associated antigen: a review of the literature. Hum Reprod 1989;4:1-12.

46. Menon U, Ryan A, Kalsi J, Gentry-Maharaj A, Dawnay A, Habib M, et al. Risk algorithm using serial biomarker measurements doubles the number of screen-detected cancers compared with a single-threshold rule in the United Kingdom Collaborative Trial of Ovarian Cancer Screening. J Clin Oncol 2015;33:2062-71.

47. Neal RD, Tharmanathan P, France B, Din NU, Cotton S, FallonFerguson J, et al. Is increased time to diagnosis and treatment in symptomatic cancer associated with poorer outcomes? systematic review. Br J Cancer 2015;112:S92-107. 